\title{
BELA LUGOSI - EIN LIEBHABER, EIN DILETTANT
}

\author{
HARUN MAYE \\ Universität zu Köln, Köln, \\ Deutschland
}

I.

„I am Dracula“. So lautete der erste Satz, den ein Vampir in einem amerikanischen Tonfilm sagen mußte, und der auch seinen Darsteller unsterblich machen sollte. Bei dem Namen Dracula bedürfen wir weder der Anschauung noch auch selbst des Bildes, sondern der Name, indem wir ihn verstehen, ist die bildlose einfache Vorstellung einer Urszene, die 1897 zum ersten Mal als Roman erschienen, und dank dem Medienverbund zwischen Phonograph, Schreibmaschine, Hypnose, Stenographie und der Sekretärin Mina Harker schon im Roman selbst technisch reproduzierbar geworden ist. ${ }^{1}$ Aber ausgerechnet über ein Medium, das im Roman nicht erwähnt wird, sollte Draculas Wiederauferstehung seitdem Nacht für Nacht laufen.

Weil Vampire und Gespenster Wiedergänger sind, und im Gegensatz zu Büchern und ihren Autoren bekanntlich nicht sterben können, müssen sie sich neue Körper und Medien suchen, in die sie fahren können. Der Journalist Abraham Stoker hat den Nachruhm und die Wertschätzung seines Namens zusammen mit dem Medium eingebüßt, das ihn berühmt gemacht hatte, nur damit fortan der Name seines schattenlosen Titelhelden für immer als belichteter Schatten über Kinoleinwände geistern konnte. Aber Spielfilme kennen so wenig originale Schöpfersubjekte wie Individuen. Dracula ist eben bloß ein Name, und als solcher ein ,,individuelles Allgemeines“ wie die Bezeichnung der Goethezeit, jener Epoche und Dichtungskonzeption, der auch Dracula poetologisch noch angehörte, für sogenannte Individuen und Phantome gleichermaßen lautete. Es ist in diesem Namen, daß wir Bela Lugosi denken. ${ }^{2}$

II.

Seit Tod Brownings Dracula von 1931 braucht dieser Name um vorstellbar zu sein, nicht mehr verstanden werden, weil er selbst angefangen hatte, sich vorzustellen. Zu Zeiten Stokers lernten die Bilder laufen, zu Zeiten Lugosis brauchten die auf Phonograph gespeicherten Stimmen des Romanpersonals nur noch mit

Hungarian Studies 14/2 (2000)

0236-6568/2000/\$5.00 CC 2000 Akadémiai Kiadó, Budapest 
den Kamerabildern synchronisiert zu werden, damit der Graf sein ,ich bin der ich-bin-da“" zitieren und also sagen konnte: „I am Dracula“. Dieser Name als tönendes Wort verschwand nicht mehr zwischen Buchdeckeln und in der Zeit, vielmehr wurde die Rede des Vampirs, wie sie gewesen ist, unsterblich. Solange Dracula eine bilderlose einfache Vorstellung in einem Vampirroman der schwarzen Romantik (Mario Praz) war, konnte er noch wahlweise als das Unbewußte, das Andere oder als Personifikation des eigenen Kolonialismus aus „dem tiefen Schacht des Ich" (Hegel) seiner westeuropäischen und amerikanischen Leser hervorgebracht werden. Erst Bela Lugosi und der Hollywood-Film haben dem Namen mit den vielen Masken ein unverwechselbares Gesicht gegeben: „Let me admit with no apology that to me Dracula is Bela Lugosi, and Lugosi is Dracula. There is no separation of the two". ${ }^{3}$ Der Doppelgänger seiner Leser ist zu einem einzigen Schauspieler geworden, und die Wunschkonstellationen von Zuschauern und Produzenten richten sich nicht mehr an bilderlose Namen, sondern direkt auf die Schauspieler und das Universal ${ }^{4}$-Medium Film. Im Imaginären des Films wird das Unbewußte von Namen und Zuschauern unmittelbar in Medientechniken übersetzbar. Rückblenden, Großaufnahmen, Zoom, Totale, Kamerafahrt und Schnitt vertreten und manipulieren Assoziation und Aufmerksamkeitsselektion. Im Film hören Namen auf bloße Vorstellungen zu sein, und fortan konnte Dracula auch im Tageslicht des Realen seine Opfer heimsuchen.

Seitdem wurde der damals fast fünfzigjährige Lugosi seinen Wiedergänger nicht mehr los. Anstelle des romantischen Liebhabers, ${ }^{5}$ den er so gerne auf der Theaterbühne gegeben hatte, aber niemals in Hollywood darstellen durfte, waren die Monster getreten. Wunschprojekte Lugosis wie eine Faust-Verfilmung oder Cyrano de Bergerac kamen über Probeaufnahmen nie hinaus. Der 'Dracula-Fluch', ${ }^{6}$ wie Lugosi die neue Machtkonstellation des Genrekinos für sich benannte, befahl ihm regelmäßig auf der Leinwand zu sterben, nur um im nächsten Film wieder von den Toten auferstehen zu müssen. Die Rolle, die ihn unsterblich machte, hat ihn auch buchstäblich in das Grab gebracht. Ob er diese Form der Unsterblichkeit wirklich geliebt hat, ist nicht sicher. Fest steht aber, daß Bela Lugosi auch als Monster eigentlich immer ein Liebhaber geblieben ist. Nicht nur erscheint er in vielen seiner Filme als „,bösartige Karikatur des romantischen Liebhabers europäischer Herkunft", ${ }^{\prime}$ sondern er hat sich vor allem selbst liebhaberisch - oder eben auch: ohne sein Metier wirklich zu beherrschen - seiner Profession gewidmet.

III.

Obwohl er für einige Jahre am Ungarischen Nationaltheater in Budapest gespielt hatte, besaß Lugosi keine Ausbildung als Schauspieler. Aus Liebe zum Theater brach er mit elf Jahren die Schulausbildung ab, riß von zu Hause aus, nur um 
sich einige Jahre später der Theatergruppe seines Schwagers anzuschließen. Seine ersten Bühnenauftritte in der Stadt Szabadka gerieten nicht zufällig zur Präfiguration seiner späten Film- und Bühnenauftritte zwischen 1945 und 1956:

Ich ging in den Chor, aber da ich nichts anderes gewesen war als Handarbeiter, war ich unbeholfen. Sie versuchten mir kleine Rollen in ihren Stücken zu geben, doch ich war so ungebildet, so einfältig, daß die Leute nur über mich lachten. Aber ich schnupperte Bühnenluft. Und ich kostete auch den ranzigen Geschmack von Demütigung. ${ }^{8}$

Wie Goethes Wilhelm Meister reiste Béla Ferenc Dezsö Blaskó, wie der spätere Dracula-Darsteller mit bürgerlichem Namen hieß, mal unter dem Namen Géza Lugosi oder Dezsö Lugosi mit einer Wanderbühne durch Ungarn und Rumänien. Schon bald nahm er dann endgültig den Künstlernamen Bela Lugosi an, den er auch gerne als Lugossy buchstabierte, da das 'y' adlige Herkunft signalisiert. Dazu passend behauptete er in Hollywood, sein Vater sei ein ungarischer Baron gewesen, um den gleichsam naturgemäß aristokratischen Anspruch auf seine DraculaInterpretation zu unterstreichen. Für die Star-Filmproduktionsgesellschaft in Ungarn spielte er unter dem Pseudonym Arisztid, was direkt 'Aristokrat' bedeutet. Mit anderen Worten: er war ein Hochstapler und Dilettant, was ja schon etymologisch nichts anderes bedeutet, als eben ein Liebhaber zu sein. Das italienische dilettante (abgeleitet vom lateinischen delecto, delectare $=$ sich oder jemanden „erfreuen“) bezeichnet ursprünglich den Liebhaber (einer Kunst) aus reinem Genuß und Unterhaltung. Der sich liebhaberisch an allen Künsten und Wissenschaften beteiligende Dilettant war ein Bildungsideal der europäischen Oberschicht bis ins 18. Jahrhundert. ${ }^{9}$ Adelige Beteiligung an höfischer Kunstproduktion oder auch an den literarischen Gesellschaften des 17. Jahrhunderts sind daher die bekanntesten Erscheinungsformen des Dilettanten. Dieser alteuropäische Dilettant war ein normaler Künstlertypus, der sowohl in der Rezeption als auch durch seine Produktionen am Kunstbetrieb ganz selbstverständlich teilnahm. Seine Nachahmungen fielen als solche nicht besonders auf, weil die gesamte Kunstproduktion und Poetik der vormodernen Zeit auf mimetischen Verfahren basierte.

Erst mit der Ausdifferenzierung eines autonomen bürgerlichen Kunstsystems wird der Dilettant zum Gegenstand von Kritik und soll aus dem Kunstsystem ausgeschlossen werden. Die neue Autonomie- und Genieästhetik setzt auf strikte Trennung: „sachlich: Trennung von Produktion und Rezeption, Schreiben und Lesen; zeitlich: Trennung von Primärem und Sekundärem; sozial: Trennung von Profis auf der einen und Laien-Publikum auf der anderen Seite. Was diese Ordnung verletzt, wird als Dilettantismus abgetan" ${ }^{10}{ }^{10}$ Erst durch diese tiefgreifenden Veränderungen im europäischen Kunstsystem bekommt der Dilettant seine pejorative Bedeutung als 'Pfuscher', 'Lügner' oder 'Stümper', und erscheint da- 
mit als Bastard, als illegitimer Bruder des (jetzt) professionell ausgebildeten Künstlers, der sich auch etymologisch als 'Kenner' und 'Könner' ausweist. Der Dilettant war von einem kultivierten Amateur und gern gesehenen Teilnehmer am Kunstbetrieb selber zu einem Monster geworden. Nicht umsonst weicht das adelige Liebesideal mit seiner Betonung der Momente von Verführung und Genuß stark von den bürgerlichen Tugendidealen ab - erst recht bei einem transsylvanischen Fürsten, der als Vlad Tepes seine Perversion schon im Namen trägt. ${ }^{11}$

In Goethes und Schillers Entwurf zu einem Schema Über den Dilettantismus (wie man seitdem erst sagt) hat die neue Kritik an dem Dilettanten ihre Programmschrift bekommen. Bezeichnenderweise wird dort ausgerechnet der dilettantische Schauspieler als „schlimmster Fall“ des Dilettantismus bestimmt: „Ueberall, wo die Kunst selbst noch kein rechtes Regulativ hat, wie in der Poesie, Gartenkunst, Schauspielkunst, richtet der Dilettantism mehr Schaden an und wird anmaßender. Der schlimmste Fall ist bei der Schauspielkunst". ${ }^{12}$ Die Schauspielkunst ist besonders anfällig für dilettantische Verfahren, weil dort das Verhältnis von Ursache und Wirkung nicht eindeutig zu bestimmen ist:

\footnotetext{
Weil der Dilettant seinen Beruf zum Selbstproducieren erst aus der Wirkung der Kunstwerke auf sich empfängt, so verwechselt er diese Wirkungen mit den objektiven Ursachen und Motiven, und meint nun den Empfindungszustand in den er versetzt ist auch produktiv und praktisch zu machen, wie wenn man mit dem Geruch einer Blume die Blume selbst hervorzubringen gedächte. ${ }^{13}$
}

Der Dilettant scheint im Schauspieler zu sich selbst zu kommen, weil beide ihre Kunstproduktion auf Nachahmung gründen. In der Tat sind die „objektiven Ursachen und Motive" der Nachahmungen des Schauspielers schwer zu bestimmen, denn direkt sichtbar ist nur deren Wirkung. Indem der Dilettant, insofern er Schauspieler ist, durch Nachahmung etwas anderes darstellen soll, als er selbst empfindet, verdoppelt er nicht nur das Problem der Nachahmung, sondern droht die Unterscheidung zwischen Künstler und Dilettant selbst ad absurdum zu führen. Das macht den Schauspieler wie den Dilettanten nicht nur grundsätzlich verdächtig, sondern zum Problemfall der goethezeitlichen Kunsttheorie.

Der Schauspieler ist eine zentrale „Reflexionsfigur“ für die zeitgleichen Debatten um Originalität und Naivität als den neuen ästhetischem Idealen in der Kunst. ${ }^{14}$ War in der gelehrten Tradition die simplicité noch selbstverständlich ein rhetorisches Prinzip und eine Tugend in Kunst und Rede, so sollte die neu konzipierte Naivität natürlich sein, und frei von aller Künstlichkeit und rhetorischer Technik. Das Naive soll aus einer natürlichen Seele kommen, und in Gedanken, Manieren, der Sprache und des Körpers, kurz: überall zum Ausdruck kommen. Der naive Ausdruck in der Kunst sollte für die Wahrheit, Schönheit und Aufrich- 
tigkeit der Gedanken einstehen. Für die Schauspielkunst liegt darin ein unauflösbares Paradox begründet, denn wie sollen naive Ursachen und Motive an einer Kunst festgestellt werden, deren offensichtliche Verfahren Nachahmung und Simulation sind:

Daß aus dem Munde des Nachahmers die Herzenssprache vernommen und seine Gebärdung als natürlich-unmittelbare Aktion begriffen werden kann, ist nur dann einsichtig, wenn man - anders als das bekannte Diderotsche Paradox, welches die Abwesenheit von Empfindung zur Voraussetzung ihrer Darstellbarkeit erklärt - einen wahrhaftig empfindenden und erlebenden Akteur vorsieht. ${ }^{15}$

Die Paradoxie der Nachahmung darf demnach nicht reflektiert werden. Vielmehr muß der professionelle Schauspieler jede Künstlichkeit und bewußte Reflexion auf seine Tätigkeit dissimulieren, damit seine Simulation von Natürlichkeit gelingen kann. Der dilettantische Schauspieler ist dazu nicht in der Lage. Ihm merkt man das 'Gewollte' und die Künstlichkeit seiner Handlungen immer an. Er ist in mehrfacher Hinsicht ungeschickt, und das ist Naivität in einem sehr trivialen Sinne.

\section{IV.}

Obwohl diese Unterscheidung nicht funktioniert, nicht funktionieren kann, war sie mehr als erfolgreich, und bestimmt noch heute das Bild vom Schauspieler. Aber auch die Probleme sind geblieben: „Ueberall, wo die Kunst selbst noch kein rechtes Regulativ hat" (Goethe/Schiller) erweist sich der Dilettant als unabweisbar, als eingeschlossener ausgeschlossener Dritter. ${ }^{16}$ Vor allem am Anfang, wenn eine Kunstform noch neu ist, sich überhaupt erst als Kunst etablieren muß, darf es nur wenige Standards geben, damit Innovationen möglich werden. Noch weiß niemand so genau was funktioniert und was nicht. Dieses Nicht-Wissen ist eine Lücke im System, die eine Unentscheidbarkeit zwischen wirklicher Innovation und Dilettantismus erzeugt.

1931 fielen in Dracula zwei Innovationen zusammen, von denen man noch nicht wußte ob sie funktionieren würden: Der Tonfilm und der erste amerikanische Horrorfilm mit einer 'übernatürlichen' Kreatur. Daß der dilettantische Schauspieler Bela Lugosi einzig in der Rolle des Grafen Dracula Erfolg haben konnte, war, so gesehen, alles andere als Zufall. Der Horror ist kein naives Genre. Um aber überhaupt erst einmal in diese 'Lücke' eindringen und im professionellen Hollywood bestehen zu können, mußte Lugosi fingieren. Also erzählte er allen, die ihn nach seinem Werdegang befragten, ,rather grim tales from my home castle in Transylvania“: 
Aus Publicitygründen, um zu vereinfachen, habe ich es stets für besser erachtet, eine Lüge über die frühen Jahre meines Lebens zu verbreiten. Ich habe immer erzählt, ich sei auf die übliche Weise zu Ungarns Royal National Theater gestoßen. Ich habe immer erzählt, ich hätte höhere Schulen, Universitäten, die Akademie der Theaterkünste, das Gymnasium in Budapest besucht. Mit Lügen zu prahlen ist vielleicht die Tollheit junger Menschen, wo die geistige Reife fehlt zu erkennen, daß die Wahrheit zu sagen manchmal wirklich achtbarer ist. ${ }^{17}$

Manchmal ist aber eine Lüge der notwendige Anfang einer Karriere. Das Spiel mit falschen Namen beherrschte er ja schon gut, als ein Darsteller für den Namen mit den vielen Masken gesucht wurde. Lon Chaney, der berühmte StummfilmHorrorstar und „Mann mit den 1000 Gesichtern“ war 1930 gerade gestorben, und obwohl erst alle anderen möglichen Schauspieler und sogar Verwandte der Produzenten getestet worden waren, fand sich niemand, der die Rolle spielen konnte. Erst dann probierte man es mit Lugosi, der die Rolle schon seit einigen Jahren erfolgreich auf der Bühne spielte. Aber weil er die Schauspielkunst nur als Autodidakt, mit einer Faszination für große Gesten und pathetische Mimik sich angeeignet hatte, konnte er den Dracula im Film, wo im Gegenteil eine Zurücknahme aller Bühnentheatralik gefordert war, nur hoffnungslos überspielen. Und genau darin lag sein überraschender Erfolg, wie auch das schnelle Ende seiner 'Karriere'.

Schon in The Silent Command (1923), Lugosis erstem amerikanischen Film, waren alle Komponenten der dilettantischen Künstlichkeit sichtbar, die sein Spiel auszeichneten:

This film revealed early trade-mark Lugosi mannerisms which became familiar in horror films particulary in closeups of the piercing eyes, menacing hand movements and the smooth way in which Bela smoked cigars. [...] Lugosi's destiny in wicked roles was fixed in this first American film, a preliminary for the characterization that would follow him to his grave. ${ }^{18}$

Manierismus war in der Tat schon immer das pejorative Markenzeichen des dilettantischen Schauspielers und eine Abweichung, die zeitgleich auf der Theaterbühne oder in einem anderen Genre negativ beurteilt wurde. ${ }^{19}$ Für den Erfolg von Dracula war Lugosis Inkompetenz mit dem Medium und der Darstellung jedoch die entscheidende Voraussetzung. Das schlechte Englisch, die zusätzliche Betonung langgezogener Vokale, die pathetischen Blicke, die kinderhafte Begeisterung für Grimassen und weit ausgestreckte, verkrampfte Hände: all das waren unvorhersehbare Neuerungen für ein Filmmonster, das zum ersten Mal keine häßlichen Masken und verkrüppelte Gliedmaßen vorzeigte (wie Chaney diese Rollen 
geprägt hatte), sondern ganz ohne unheimliche Kostüme, lange Zähne, Schminke und Blut einfach nur im Smoking auftrat. Was in späteren Rollen - wenn es nicht sowieso Komödien waren - das Publikum zum Lachen anregte, konnte in Dracula überzeugen. Ein vormoderner Liebhaber, der schon seines Blutes wegen weder arbeiten noch mit technischen Medien umgehen konnte, dessen Macht sich auf Rituale und sexuelle Anziehungskraft verlassen muß, anstelle über Geld und Wissen verfügen zu können - wer hätte diesem unzeitgemäßen Namen ein besseres Gesicht geben können als Bela Lugosi?

\title{
V.
}

Das Geheimnis seines Erfolges als Schauspieler sah der Autodidakt in einer bestimmten Technik des Selbst:

\begin{abstract}
...daß ich die Fähigkeit hatte, meinen Willen, meinen Verstand, meinen Körper, meine Gefühle in einem einzigen tiefen und strömenden Kanal zusammenzuführen [...] wenn das, was ich damals tat und noch heuet tue, Wahnsinn ist, dann laßt mich wahnsinnig sein, da ich so mein Ziel erreicht habe. ${ }^{20}$
\end{abstract}

Ganz wie sein gräfliches Vorbild sah er sich auch in der Lage durch Hypnose oder eben gleich durch Wahnsinn, alle Sinne und Daten, die ihm zur Verfügung stehen, in einem einzigen Informationsfluß bündeln und damit verstärken zu können. Aber allen Hypnotiseuren, die Lugosi im Film gespielt hatte, zum Trotz, war diese Fähigkeit allerdings weder einer natürlichen Gabe noch hypnotischen Fähigkeiten Lugosis zuzuschreiben, sondern ganz einfach der Konditionierung von autodidaktischen, d.h. passionierten und anleitungslosen Lesern durch das Medium Buch. ${ }^{21}$ Denn aller Wille, Verstand und alle Gefühle Lugosis stammen aus Büchern. Um seine kaum vorhandene Schulbildung zu kompensieren, entschloß sich der Autodidakt zu dem einzig möglichen ,,self-improvement program: „For ten years, day and night, night and day, with only one, two, three hours sleep, I read and read and read until I could talk with any college professor in the world". ${ }^{22}$ Der vermeintlich wahnsinnige Schauspieler war ganz einfach ein 'ausschweifender' Leser, der sich rühmte, täglich sechs verschiedene Zeitungen zu lesen. ${ }^{23}$ So viel typographische Informationsverarbeitung bleibt nicht ohne Folgen. Der Buchdruck hat ja nicht nur zu einem Niedergang des lauten Lesens geführt, den Leser isoliert und verstummen lassen, sondern - wie Marshall McLuhan immer wieder betont hat - vor allem durch die Linearität der Buchstaben- und Gedankenführung die Vielfalt der Sinneswahrnehmungen in den Hintergrund gedrängt und in einen einzigen Gesichtspunkt, einen Sinn gebündelt. ${ }^{24}$ Dieser „Kanal“, in dem Lugosi alle seine Sinne bündeln zu können glaubte, heißt nicht „Wahnsinn“ sondern ist 
ein „Engpaß der Signifikanten“ (Kittler), durch den alle Information fließen muß - erst recht wenn sie durch das technische Medium Druck in eine lineare Reihe gebracht wird.

Aber weil nicht nur Wahnsinnige, sondern auch Dilettanten aus dem professionellen Ablauf jedes Systems und Metiers ausgegrenzt werden, war Lugosis Erfolg nur geliehen. Durch die Systemlücke Dracula, die Hollywood notwendig nicht schließen konnte, war Lugosi nicht nur der Eintritt in eine unerreichbar scheinende Welt gelungen, sondern gleichzeitig auch jede Chance auf individuelle Veränderung genommen. The Mark of the Vampire, wie sein nächster Film mit dem Dracula-Regisseur Tod Browning heißen sollte, hat ihn nie mehr losgelassen. Im Zeichen des Vampirs sollte Bela Lugosis einzig mögliche Zukunft in Hollywood verlaufen. Das Establishment hatte zurückgebissen, und den eingeschlichenen Dilettanten exakt durch die Rolle, die ihm Einlaß gewährte, als solchen enttarnt und markiert.

Nach dem Ende des Zweiten Weltkriegs und der Medienkonkurrenz mit dem Fernsehen, war Bela Lugosis kurze Karriere zu ihrem Ende gekommen. Seine alten Filme wurden mittlerweile im Fernsehen gezeigt - ein Medienwechsel, der sein unnatürliches Spiel unnatürlicher Kreaturen plötzlich lächerlich aussehen ließ. Der Film war das ,ideale Medium“ für den Horror gewesen, ganz einfach weil dem Unheimlichen der Romantik ein Gesicht gegeben wurde, und dessen psychologischen Funktionen direkt als filmtechnische Verfahren implementiert werden konnten. ${ }^{25} \mathrm{Im}$ detailarmen Fernsehformat verflüchtigten sich romantische Phantastik und Effekte des Films gleichermaßen. Wie Philip Auslander gezeigt hat, wird jeder Kinofilm, der im Fernsehen ausgestrahlt wird, zu einer Aufführung in der Gegenwart ('live performance') „remediatisiert“. ${ }^{26}$ Die Performanz des Mediums Fernsehen beruht auf der Erzeugung einer Rezeptionshaltung von Unmittelbarkeit und Natürlichkeit der gezeigten Ereignisse. Nicht nur die Nachrichtensendungen mit ihrem Anspruch auf Realität und Wahrheit, sowie eine Vielzahl von Live-Sendungen machen den Unterschied zum Film aus, sondern vor allem die Selbstinszenierung des Fernsehens als Theater:

It is my contention that this ideologically engrained sense of television
as a live medium makes its historical relationship to the theatre diffe-
rent from that of film, and enabled television to colonize liveness,
the one aspect of theatrical presentation that film could not replicate.
[...] Whereas film could only remediate the theatre at these structural
levels, television could remediate theatre at the ontological level
through its claim to immediacy. It is also significant in this context
that television not only remediates live performance, it remediates
film in a way that film has never remediated television. ${ }^{27}$

In der erzeugten Illusion von Aktualität und Unmittelbarkeit des Fernsehens, sowie dem mitaufgerufenen Kontext einer Theateraufführung, fiel Lugosi wieder 
als nicht naiver, dilettantischer Schauspieler auf, dessen Bewegungen übertrieben und Figuren überspielt waren.

Dementsprechend mußte der verarmte Schauspieler im hohen Alter und seinen letzten Tod vor Augen aus finanziellen Gründen immer noch in der (Wander-) Bühnenversion des Dracula für kleines Geld vor einem ebenfalls kleinen und gröhlenden Publikum auftreten. Der alte Mann, gehüllt in einen schwarzem Umhang, mit seinen manierierten Bewegungen, war längst zu seiner eigenen Karikatur geworden. ${ }^{28}$ Selbst in einem Nachtclub in Las Vegas gab der über 70jährige in der „Bela Lugosi Revue“ sechs Wochen lang noch jeden Abend den Dracula. Er wünschte sich sogar ein 3-D Remake in Technicolor. So sollte sich für sein Leben bewahrheiten, was er als Dracula schon zu Mina Seward gesagt hatte: ,...to die, to be really dead: that must be glorious. There are far worse things, awaiting man, than death“. In einem seiner späten Interviews wußte er bereits, daß er die letzte Ruhe wohl niemals finden würde:

- „Did the role depress you?“

- „Very much. It hunted me. I often dreamed of the dead.“

- „Doesn't Dracula ever end for you?“

- „No, no. Dracula never ends. I don't know whether I shall call it a fortunate recall, but it never ends". ${ }^{29}$

\section{Anmerkungen}

1. Zu den verschiedenen Möglichkeiten einer technischen Reproduzierbarkeit in Stokers Dracula als dem „Sachbuch unserer Bürokratisierung“ vgl. nach wie vor Friedrich Kittler: Draculas Vermächtnis. In: Ders.: Draculas Vermächtnis. Technische Schriften. Leipzig 1993. S. 11-57.

2. „Der Name ist so die Sache, wie sie im Reiche der Vorstellung vorhanden ist und Gültigkeit hat. Das $\beta ß)$ reproduzierende Gedächtnis hat und erkennt im Namen die Sache und mit der Sache den Namen, ohne Anschauung und Bild. [...] Bei dem Namen Löwe bedürfen wir weder der Anschauung eines solchen Tieres noch auch selbst des Bildes, sondern der Name, indem wir ihn verstehen, ist die bildlose einfache Vorstellung. Es ist in Namen, daß wir denken" - Georg Wilhelm Friedrich Hegel: Enzyklopädie der philosophischen Wissenschaften im Grundrisse. Dritter Teil (1830). Frankfurt/Main 1986. S. 278.

3. So die Schauspielerin und Filmpartnerin Carroll Borland in ihrem Vorwort zu Richard Bojarskis The Films of Bela Lugosi, Secaucus - N. J. 1980. S. 9.

4. Universal ist auch der Name der berühmten Filmproduktionsgesellschaft von Dracula und anderen Filmen Lugosis. Universal war das führende Filmstudio für Horrorfilme in den 20er, 30er und 40er Jahren, und prägte Stil, Sujets und Ausstattung dieses Genres bis heute. Daß der Erfolg von Dracula hauptsächlich seinem Medium zu verdanken ist, wußte auch sein Hauptdarsteller: „,...the popularity of horror pictures is understandable. The screen is the ideal medium for the presentation of gruesome tales (...) And they have an almost universal appeal" - so Bela Lugosi in einem Interview, zit. n. Richard Bojarski: The Films of Bela Lugosi, Secaucus - N. J. 1980. S. 34. 
5. Während seiner Zeit an Ungarns Nationaltheater in Budapest spielte er tatsächlich oft in klassisch-romantischen Dramen, allerdings, bis auf den Romeo in Shakespeares Romeo and Juliet und Jesus von Nazareth in der Passion, immer nur in Nebenrollen.

6. „Where once I had been the master of my professional destinies, with a repertoire embracing all kinds and types of men, from Romeo to the classics of Ibsen and Rostand, I became Dracula's puppet $[. .$.$] The shadowy figure of Dracula, more than any casting office, dictated the kind of$ parts I played [...] never, surely has a role so influenced and dominated an actor's personal life and private fortunes", so Lugosi in einem Interview, zit. n. Robert Cremer: Lugosi. The Man behind the Cape. Chicago 1976.

7. Hans Scheugl: Sexualität und Neurose im Film. Die Kinomythen von Griffith bis Warhol. München 1974.

8. Bela Lugosi in einem Interview mit Gladys Hall (Memoes of a Madman, Juli 1941), zit. nach Rolf Giesen: Lexikon des phantastischen Films. Bd. 2. Frankfurt/Main - Berlin - Wien 1984. S. 55.

9. Zur Etymologie und Sachgeschichte des Dilettantismus vgl. Georg Stanitzek: Dilettant, in: Reallexikon der deutschen Literaturwissenschaft. Hrsg. v. Klaus Weimar. Bd. 1. Berlin - New York 1997. S. 364-366.

10. Georg Stanitzek: Über Professionalität. In: Mitteilungen des Deutschen Germanistenverbandes 43, Heft 4 (1996). S. 19.

11. 'Vlad Tepes' ist der Name des Rumänischen Fürsten, der die Walachei von 1456-1462 regierte und der das historische Vorbild für Bram Stokers Dracula war. Sein Name bedeutet „Vlad der Pfähler" und leitet sich von dessen Vorliebe für eine Foltermethode her, bei der ein Pfahl langsam durch den Anus in den Leib eines Opfers eindrang. Zu dem historischen Hintergrund vgl. Ralf-Peter Märtin: Dracula. Das Leben des Fürsten Vlad Tepes. Frankfurt/Main 1991.

12. Johann Wolfgang Goethe und Friedrich Schiller: Über den Dilettantismus. In: Schillers Werke. Nationalausgabe. Bd. 21. Hrsg. v. Benno von Wiese. Weimar 1963. S. 61.

13. Ebenda. S. 60. Gegen diesen schädlichen Trieb des Dilettanten „oder eigentlich Pfuscher“ über die Nachahmung von Gefühlen oder Stimmungen zur Nachahmung von Kunstwerken zu gelangen, gibt es laut Goethe und Schiller nur ein Remedium: „Allgemeine Grundsätze“ und „,strengste Regeln" für die Kunst. Hier wird besonders deutlich, daß die positive Selbstbeschreibung des Klassizismus in Deutschland eigentlich negative Beweggründe und Ursachen hat: die neuen, strengen Regeln der Kunst haben in erster Linie die Funktion einer Diskurspolizei und dienen u.a. einer Abwehr des Dilettantismus.

14. Ausführlich dazu Ursula Geitner: Menschen als Schauspieler und Schauspieler als Menschen. In: Dies.: Die Sprache der Verstellung. Studien zum rhetorischen und anthropologischen Wissen im 17. Und 18. Jahrhundert. Tübingen 1992. S. 284-343.

15. Ebenda. S. 286. Die klassische Studie zu diesem Problem von Naivität und Bewußtsein in der Kunst ist nach wie vor Heinrich von Kleists Über das Marionettentheater.

16. Vgl. hierzu Erhard Schüttpelz: Die Akademie der Dilettanten. (Back to D.). In: Akademie. Katalog. Hrsg. v. Stephan Dillemuth. Köln 1995. S. 40-57.

17. Bela Lugosi in einem Interview mit Gladys Hall (Memoes of a Madman, Juli 1941), zit. nach Rolf Giesen: Lexikon des phantastischen Films. Bd. 2. Frankfurt/Main - Berlin - Wien 1984. S. 53.

18. Richard Bojarski: The Films of Bela Lugosi. Secaucus, N.J. 1980. S. 22.

19. In einer Variety-Kritik zu Arabesque, dem ersten Brodway-Stück Lugosis, wird dessen Darstellung eines arabischen Scheichs aus genau diesen Gründen kritisiert: „The Sheik is overplayed and underdone by Bela Lugosi, but [at least] he gets the idea across", zit. n. Ebenda. S. 23.

20. Bela Lugosi in einem Interview mit Gladys Hall (Memoes of a Madman, Juli 1941), zit. nach 
Rolf Giesen: Lexikon des phantastischen Films. Bd. 2. Frankfurt/Main - Berlin - Wien 1984. S. 55.

21. Daß der Buchdruck als technisches Medium auch einem scheinbar illegitimen Umgang mit Wissen förderlich werden konnte, hatte schon Joseph von Eichendorff bemerkt. Anhand von Büchern konnten ungebildete Leser plötzlich in jeder Form von Wissen herumblättern: „Durch den Druck ist aber in der Tat die ganze Literatur ein Buch geworden, in welchem jeder nach Belieben blättern mag und daraus ein allgemeiner Dilettantismus der Produzenten wie der Konsumenten entstanden“ - Joseph von Eichendorff: Geschichte der poetischen Literatur Deutschlands. In: Ders.: Werke. Bd. 3. München 1976. S. 605. Zit. n. Georg Stanitzek: Über Professionalität. In: Mitteilungen des deutschen Germanistenverbandes 43 (1996). S. 22.

22. Lugosi zit. n. Richard Bojarski: The Films of Bela Lugosi. Secaucus, N. J. 1980. S. 15

23. „His hobbies consisted of building up his stamp collection, hosting Hungarian-style parties at his home and spending hours „dry reading“ in his extensive library of subjects such as politics, history, economy, social revolution, religion and philosophy. A liberal democrat, Lugosi read up to six newspapers daily to stay abreast of current events“. Ebenda. S. 37.

24. z. B. Marshall McLuhan: Die Gutenberg-Galaxis. In: Ders.: Medien verstehen. Der McLuhanReader. Hrsg. v. Martin Baltes u.a. Mannheim 1997. S. 85-86. McLuhan zitiert in diesem Zusammenhang aus William Ivins Studie Prints and Visual Communications, der wie Lugosi für das Phänomen die technische Metapher des „Kanals“ gebraucht: ,je genauer wir unsere Daten bei einer Beweisführung auf die Daten beschränken, die uns durch ein und denselben Sinneskanal zukommen, desto weniger neigen wir dazu, in unserem Gedankengang Fehler zu machen“. Ebenda. Der Leser bekommt demnach alle Informationen aus einem 'Gesichtspunkt', aus einem 'Kanal', eben aus einem Buch übermittelt, und lernt dementsprechend auch selber in seiner Produktion alle Daten unter einem Gesichtspunkt zu präsentieren und durch einen Kanal fließen zu lassen.

25. Dazu immer noch überzeugend Friedrich Kittler: Grammophon Film Typewriter. Berlin 1986. S. 214-255.

26. Philip Auslander: Liveness. Performance in a mediatized culture. London - New York 1999. S. 10-23.

27. Ebenda. S. 13.

28. Folgerichtig begründen diese ,trade-mark Lugosi mannerisms“ auch seinen Nachruhm. Aus dem Wahnsinn (der Lektüre) entstanden, sind sie immer noch eine Parabel für „weise Idioten“ wie Osbie Feel in Pynchons Roman Gravity's Rainbow: „'Osbie, bin ich verrückt geworden?' [...] 'Na klar, na klar', macht Osbie und vollführt eine fließende Bewegung von den Fingerspitzen bis zum Handgelenk, frei nach der Geste, mit der Bela Lugosi irgendeinem Idioten von jugendlichem Helden in White Zombie das prekäre Glas voll präpariertem Wein kredenzt, im ersten Film, den Osbie je gesehen hat, und in gewisser Weise auch dem letzten, denn immer noch rangiert er, neben Son of Frankenstein und Freaks und Flying Down to Rio, ganz oben auf Osbies Liste seiner Lieblingsstreifen“- Thomas Pynchon: Die Enden der Parabel (1973). Reinbek bei Hamburg 1981. S. 173. Den Hinweis auf diese Stelle verdanke ich Markus Krajewski.

29. Bela Lugosi im Interview, zit. nach dem Dokumentarfilm Bela Lugosi. The Forgotten King [1990] von Forest J. Ackerman. 


\title{
HUNGARIAN STUDIES \\ a Journal of the International Association of Hungarian Studies \\ (Nemzetközi Magyar Filológiai Társaság)
}

\author{
Editor-in-Chief
}

Mihály Szegedy-Maszák

\section{Editors}

Richard Aczel

Gábor Bezeczky

József Jankovics

Peter Schimert

Volume 14

Numbers 1, 2

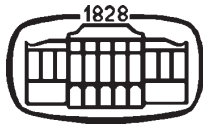

AKADÉMIAI KIADÓ, BUDAPEST

2000 


\section{CONTENTS}

Bayerle, Gustav: Formation of the Habsburg-Ottoman Frontier

in the Danubian Region: Buda, $1541 \ldots \ldots \ldots \ldots \ldots \ldots \ldots \ldots . \ldots 163$

Bielasiak, Jack: Poland's Transition as Political Repolarization . . . . . . . 259

Bischof, Günter: Restoration, not Renewal: From Nazi to Four-Power

Occupation - The Difficult Transition to Democracy in Austria

after $1945 \ldots \ldots \ldots \ldots \ldots \ldots$. . . . . . . . . . . . . . . . . 207

Borhi, László: Some Questions on Hungarian-Soviet Relations, 1949-1955 . . . . . . . . . . . . . . . . . . . . . . . . . 1

Bozóki, András: The Roundtable Talks of 1989: Participants, Political Visions, and Historical References . . . . . . . . . . . . 241

Cooper, Thomas: Dezső Kosztolányi and Intertextuality. Anticipations

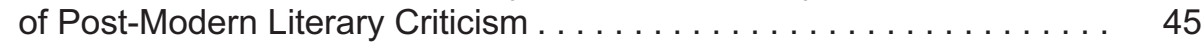

Dombrowsky, Mirko: Zu Péter Lengyels Cobblestone. . . . . . . . . . . . 121

Frank, Tibor: The Austro-Hungarian Compromise of 1867 and Its Contemporary Critics . . . . . . . . . . . . . . . . . . . . 193

Hajdu, Péter: Metaphorische Geschichteninteraktion. Die Taube im Käfig des Kálmán Mikszáth . . . . . . . . . . . . . . . . . . 103

Kulcsár Szabó, Ernő: Aspekte des (Un)Vollkommenen. Fragment und vollendetes Kunstwerk im Horizontwandel der Sprachlichkeit . . . . . . . 85

Maye, Harun: Bela Lugosi - Ein Liebhaber, ein Dilettant . . . . . . . . . . 285

Pók, Attila: Scapegoats in Post-World War I Hungarian Political

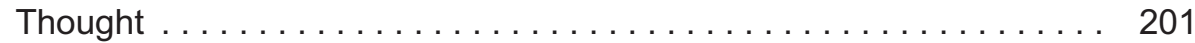

Raun, Toivo U.: The Baltic States after the Collapse of the Soviet Union . . 275

Sinor, Denis: The First Change of Regime in Hungarian History . . . . . 153

Sohár, Anikó: Thy Speech Bewrayeth Thee: Thou Shalt Not Steal the Prestige of Foreign Literatures. Pseudotranslations in Hungary

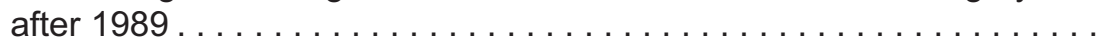

Szegedy-Maszák, Mihály: From Enlightenment Universalism to Romantic Nationalism . . . . . . . . . . . . . . . .

Tóth, István György: Alternatives in Hungarian History in the Seventeenth Century............................. 171

Tökés, Rudolf L.: Revolution Reconsidered: Institution-Building

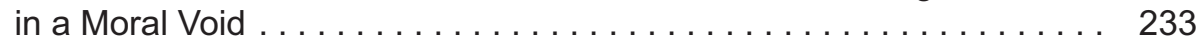

Zirkuli, Péter: Mythes des origines et hypothèse linguistique chez Sándor

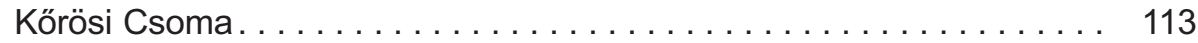




\section{REVIEWS}

In Quest of the 'Miracle Stag': The Poetry of Hungary. An Anthology of Hungarian Poetry from the 13th Century to the Present in English Translation. Vol. I (Adam Makkai ed.) (Richard Aczel, Christof Scheele,

Thomas E. Cooper, Mihály Szegedy-Maszák) . . . . . . . . . . . . . 127

Der literaturgeschichtliche Fahrplan (Kristóf Szabó) . . . . . . . . . . . . 139

The Kiss: 20th Century Hungarian Short Stories (István Bart ed.)

(Frank J. Kearful) . . . . . . . . . . . . . . . . . . . . . . . . . . . 142

A Cultural History of Hungary (László Kósa ed.) (James T. Wilson) . . . . . . 147

Gábor Tolcsvai-Nagy: A magyar nyelv stilisztikája [Hungarian Stylistics]

(Laura Knudsen) . . . . . . . . . . . . . . . . . . . . . . . 150 